\title{
La biodiversité dans des territoires insulaires, approche théorique et perspectives de développement
}

Biodiversity in island territories, theoretical approach and development prospects

Jean-Raphaël Gros-Désormeaux

\section{OpenEdition}

\section{Journals}

Édition électronique

URL : http://journals.openedition.org/developpementdurable/9241

DOI : 10.4000/developpementdurable.9241

ISSN : 1772-9971

Éditeur

Association DD\&T

Référence électronique

Jean-Raphaël Gros-Désormeaux, «La biodiversité dans des territoires insulaires, approche théorique et perspectives de développement », Développement durable et territoires [En ligne], Vol. 3, n 1 | Mai 2012, mis en ligne le 24 mai 2012, consulté le 19 avril 2019. URL : http://journals.openedition.org/ developpementdurable/9241; DOI : 10.4000/developpementdurable.9241

Ce document a été généré automatiquement le 19 avril 2019

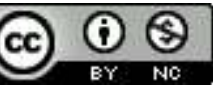

Développement Durable et Territoires est mis à disposition selon les termes de la licence Creative Commons Attribution - Pas d'Utilisation Commerciale 4.0 International. 


\title{
La biodiversité dans des territoires insulaires, approche théorique et perspectives de développement
}

\author{
Biodiversity in island territories, theoretical approach and development \\ prospects
}

Jean-Raphaël Gros-Désormeaux

Il aura fallu moins d'un siècle pour que les sociétés occidentales modifient leur perception du monde vivant et plus largement de la nature. La volonté de maîtriser une nature anciennement considérée comme hostile et répulsive a fait place à une démarche beaucoup plus respectueuse de la vie en cherchant un équilibre entre la satisfaction des besoins de l'humanité et la nécessité de ne pas détruire la diversité du monde vivant. Issu de ce changement de perception, le concept de biodiversité a été consacré lors de la Conférence sur l'environnement et le développement à Rio de Janeiro en juin 1992. Cet évènement a mis l'accent sur la nécessité de protéger la biosphère tout en assurant un développement durable. Cette sphère biologique est indissociable du concept de biodiversité : elle contient l'extraordinaire complexité structurale et organisationnelle du vivant. À l'image d'un organisme biologique, les entités qui la composent entretiennent les relations responsables des grands équilibres biologiques de la terre. À l'échelle des temps géologiques, les taxa se sont succédés dans une biosphère qu'ils ont su se partager grâce à leur adaptabilité. L'espèce humaine est fréquemment accusée dans nombre d'évènements d'extinction. Du fait de la dynamique de sa population, l'accroissement de ses besoins en espace et en ressources naturelles menace l'existence de l'actuelle biosphère par d'irrémédiables changements dans la composition et la structuration de sa diversité biologique. Le taux d'extinction naturel a été estimé à deux pour mille espèces par an (Le Danff, 2002). Or, la constitution d'une nouvelle espèce prendrait entre cinq et dix millions d'années (Leakey et al., 1998). Depuis 1970, cinquante-huit espèces de poissons, une espèce de mammifère et neuf espèces d'oiseaux se seraient éteintes (PNUE, 2002). Des prospectives sur l'impact des changements climatiques estiment qu'un réchauffement global de $0,8^{\circ} \mathrm{C}$ à $2,2^{\circ} \mathrm{C}$ en 2050 devrait se solder à terme par l'extinction 
de $15 \%$ à $37 \%$ des espèces de papillons, vertébrés et plantes terrestres peuplant les diverses grandes régions considérées (Teyssèdre, 2005). Cette disparition massive et rapide d'espèces, qualifiée par certains de sixième extinction (Leakey et al., 1998; Teyssèdre, 2005), est caractéristique du phénomène d'érosion de la diversité biologique.

Conscient qu'il s'intègre à un ensemble dont l'estimation irait de trois à cent millions d'espèces constitutives de la diversité de la biosphère, l'homme met en œuvre des procédures de protection et de conservation d'écosystèmes qu'il juge remarquables, en se fondant sur le savoir et les pratiques autours desquels s'organisent sa société. Dès lors, afin d'apporter des justifications à cette démarche, le concept de biodiversité sera approché dans une dimension élargie. Il tend à devenir une véritable idéologie insistant sur la dépendance des sociétés humaines à l'égard de la diversité biologique à travers des problématiques aussi variées que l'agriculture, l'élevage, la pêche, les forêts, la cueillette, la pharmacie, les cosmétiques, l'alimentation, l'industrie, etc. La notion de biodiversité s'applique de plus en plus à tout ce qui relève des relations entre l'homme et la nature. L'adoption de cette notion, ne peut-elle pas alors s'envisager comme un simple changement de vocable, pour parler des relations entre l'homme et la nature, témoignant d'une volonté humaine d'établir des rapports respectueux vis-à-vis des autres espèces avec lesquelles la société partage la biosphère? Ainsi, la notion de biodiversité sera interprétée différemment selon les groupes sociaux en présence: biologistes, agriculteurs, industriels, écologues et écologistes conçoivent la biodiversité en fonction de leurs propres préoccupations. Certains puristes sont d'avis que l'utilisation du terme biodiversité doit se limiter à son acception originelle, soit la quantification et la description du monde vivant sous toutes ses formes et à toutes ses échelles d'intégration biologique. Ils reconnaissent néanmoins que la médiatisation et la popularisation du concept nécessitent de mener une réflexion dont les limites sont d'ores et déjà imposées par la diversité des groupes d'êtres vivants et des complexes écologiques, mais bien plus encore par sa dimension transdisciplinaire. Ainsi, nous nous proposons d'approcher la problématique de la biodiversité dans les territoires insulaires en nous intéressant plus particulièrement à l'une des classes zoologiques les plus diversifiées dans l'un des territoires insulaires les plus remarquables, de l'une des régions actuellement qualifiées de hauts lieux de la diversité biologique mondiale : l'avifaune caribéenne à la Martinique.

\section{Les enjeux de la diversité biologique insulaire, un endémisme menacé}

3 La gestion de la diversité biologique est un objectif prioritaire pour les espaces insulaires. Depuis les travaux de Von Humboldt, Darwin et Wallace, les îles sont présentées comme des écosystèmes singuliers et fragiles. L'originalité des îles est confrontée à une difficulté majeure. Lorsqu'un groupe d'organismes arrive à coloniser un système insulaire, leur descendance aura tendance à diverger de leur état originel. Les conditions d'isolement favorisent le développement d'individus dont les caractères biologiques sont les mieux adaptés à l'environnement. Sur le long terme, la vie sur l'île, singulièrement différente de celle des espaces continentaux, donne lieu à des structurations biocénotiques ${ }^{1}$ adaptées à un environnement insulaire propre. En d'autres termes, les unités biocénotiques qui ont subi l'insularité sur un temps relativement long sont condamnées à vivre dans ces conditions pour assurer leur survie : ce sont généralement des espèces qui sont dites endémiques à un territoire donné. L'endémisme est le phénomène par lequel une espèce ou 
un groupe taxonomique est strictement inféodé à une aire biogéographique donnée, généralement de surface restreinte, dans laquelle il s'est différencié par suite de l'existence de conditions écologiques spéciales propres à l'aire considérée (Ramade, 2002). Une espèce endémique est donc inféodée à une aire biogéographique généralement de faible étendue. Cette définition de l'endémisme renvoie à deux facteurs interdépendants : une genèse ou une évolution particulière des populations, conduisant à une aire de répartition limitée. L'endémisme implique que la naissance ou l'évolution du taxon s'est faite selon des modalités particulières. De manière simpliste, deux schémas sont actuellement admis. Dans le premier, l'endémisme s'expliquerait par l'extinction de populations. L'espèce, anciennement répartie sur des surfaces plus importantes, se retrouve progressivement isolée à l'intérieur de territoires limités. Ce type est qualifié de paléo-endémique (Whitteker, 1998). Dans le second cas, l'aire de distribution de l'espèce se limite à la zone où la forme a pris naissance. À cela s'ajoute le fait que le taxon ne s'est dispersé que sur des surfaces plus ou moins faibles. On parlera alors de néo-endémisme (Whitteker, 1998). Ces deux formes font ressortir l'importance de la surface occupée par l'espèce pour la définition de cette notion d'endémisme. Ainsi, le terme endémique ne peut qualifier une espèce que s'il s'applique à un espace limité et plus ou moins restreint.

Cela a favorisé l'émergence de la notion de centre d'endémisme. Ce terme est employé pour définir des régions biogéographiques ${ }^{2}$ où l'on aurait répertorié un nombre conséquent d'espèces endémiques. Or, les régions insulaires sont considérées comme des centres privilégiés d'endémisme. En effet, parmi les régions dont la diversité biologique est significative pour son taux d'endémisme, on dénombre six principales régions insulaires : la Nouvelle-Calédonie, la Nouvelle-Zélande, la Caraïbe, les îles de la Polynésie et de la Micronésie, Madagascar et les Philippines (Myers et al., 2000 ; Mittermeier et al., 2005). Le pourcentage d'endémisme en vertébrés de chacune de ces six régions est de loin supérieur à celui de l'Europe.

Tableau 1. Endémisme des vertébrés ${ }^{3}$ dans quelques régions du monde

\begin{tabular}{|l|l|l|l|}
\hline Régions & Nombre d'espèces & Nombre d'endémiques & Taux d'endémisme ${ }^{4}$ \\
\hline Madagascar & 987 & 771 & $78 \%$ \\
\hline Polynésie et Micronésie & 342 & 223 & $65 \%$ \\
\hline Nouvelle-Zélande & 217 & 136 & $63 \%$ \\
\hline Caraïbe & 1518 & 779 & $51 \%$ \\
\hline Philippines & 1093 & 518 & $47 \%$ \\
\hline Nouvelle-Calédonie & 190 & 84 & $44 \%$ \\
\hline Europe & 1063 & 254 & $24 \%$ \\
\hline
\end{tabular}

Sources : Myers et al. (2000) et Lévêque et al. (2001).

5 Les espèces endémiques, véritables spécificités des écosystèmes insulaires, sont rendues plus vulnérables à l'extinction par l'anthropisation. En effet les spécificités biologiques 
des îles sont de plus en plus fragilisées à cause d'une diminution de plus en plus forte de l'un des fondements biogéographiques de l'insularité: l'isolement (Doumenge, 1987, 1994 ; Taglioni, 2003). Le rapprochement des îles aux continents et des îles entre elles est le premier facteur de la rareté, du déclin et de l'extinction de l'endémisme.

6 En effet, la théorie de l'équilibre dynamique de MacArthur et Wilson (1967) met en évidence une forme d'instabilité relative dans l'évolution de la composition taxinomique des faunes insulaires à cause d'un renouvellement des espèces. Ce phénomène résulte d'un glissement évolutif de stratégies adaptatives.

7 La notion de stratégie adaptative (Blondel, 1979, 1986, 1995) s'utilise pour caractériser un type d'adaptation d'une population ou d'une communauté vivante à des conditions environnementales particulières (Ramade, 2002). Les stratégies démographiques, spécifiques à l'analyse des populations, sont qualifiées de type $r$ pour des espèces qui se rencontrent de préférence dans des écosystèmes juvéniles. Le type $k$ est utilisé pour les espèces des écosystèmes à maturité. L'espèce se situe sur un continuum entre les deux stratégies extrêmes. Dans l'évolution des taxa qui sont soumis aux conditions d'insularité, le glissement évolutif se traduit par les réponses adaptatives, soit les symptômes du syndrome d'insularité (Blondel, 1995). Les taxa qui se développent en situation d'insularité sont des colonisateurs affichant une sélection de type $r$ qui progressivement tendront à se transformer en sédentaires dont la sélection sera de type $k$. En effet, les meilleurs candidats à la colonisation sont des espèces à tendance généraliste (type $r$ ), alors que les espèces qui présentent les caractéristiques intrinsèques optimales pour l'exploitation des ressources insulaires sont des espèces plus spécialisées (type $k$ ). Un taxon est dit "généraliste» dès lors qu'il n'a pas d'exigences écologiques précises. A contrario, les spécialistes ont des exigences de survie plus contraignantes. Ce sont généralement des espèces qui sont inféodées à des habitats limités. La mise en évidence d'un glissement évolutif se fonde sur les travaux menés par Diamond, à la fin des années 1960, sur l'avifaune des îles Channel en Californie, qui furent largement discutés par Johnson, Lynch et Lack dans les années 70 (Blondel, 1986). En prenant pour modèle l'avifaune des Petites Antilles, Ricklefs $(1972,2002,2004)$ a proposé quatre stades successifs décrivant l'évolution des communautés insulaires. Le premier stade évolutif se caractérise par des communautés dont les organismes sont morphologiquement, démographiquement ou encore éthologiquement similaires à leurs homologues continentaux. Ils peuvent théoriquement se retrouver sur l'ensemble de la région insulaire. $\mathrm{Au}$ second stade, les taxa ne sont plus présents sur l'ensemble de la région insulaire. À cette échelle, ils présentent des différenciations de caractères selon l'île occupée. Au stade suivant, les taxa sont représentés par des sous-espèces présentes dans quelques îles. Enfin, au dernier stade évolutif, la spécialisation des taxa est telle qu'ils deviennent endémiques au territoire. Leur répartition se limite généralement à une île. Malgré leur haute spécialisation à l'environnement insulaire dans lequel le taxon s'est différencié, les espèces endémiques sont susceptibles d'être extrêmement vulnérables à l'extinction. Cela s'explique par la supériorité compétitive des espèces invasives qui contraindront les endémiques à se cantonner progressivement à des territoires de moins en moins importants.

Dès lors qu'un taxon s'installe sur une île sur un temps géologique plus ou moins important, il se spécialise dans l'exploitation la plus optimale des ressources offertes par son environnement (MacArthur et al., 1967). Les pressions exercées par ce dernier tendent à le faire progressivement diverger de sa forme originelle. Le taxon résultant de ce 
mécanisme de spéciation générateur d'endémiques, se traduit par l'acquisition de spécialisations de plus en plus poussées à l'échelle évolutive. Bien que la colonisation successive d'îles au sein d'un archipel soit avérée, ce niveau de spécialisation est tel qu'il devient difficile, voire même impossible pour ce dernier de recoloniser un espace continental. La réduction de la compétition et de la prédation en situation d'insularité entraîne sur le long terme un amoindrissement des capacités de défense et de dispersion. Les organismes insulaires les plus anciens atteignent donc un tel degré de spécialisation qu'ils deviennent, malgré l'adaptation à leur environnement insulaire, extrêmement vulnérables aux invasions. En effet, leur infériorité compétitive les rend impuissants face aux compétiteurs, aux prédateurs et aux parasites exogènes. Sur le long terme, les invasifs peuvent entrainer l'extinction de l'espèce autochtone. Or, selon la théorie de l'équilibre dynamique (MacArthur et al., 1967), les évènements de colonisation se répètent dans le temps. Cela suppose des successions d'épisodes de colonisation, de spécialisation et d'extinction résultant de phases d'expansion puis de contraction des populations : ce sont les cycles de taxons (Blondel, 1995 ; Ricklefs et al., 2002). En fonction de facteurs tels les changements climatiques globaux et les évènements d'invasion (Whittaker, 1998), le cycle se répète dans des intervalles de temps plus ou moins importants selon le degré d'isolement de l'île et le pouvoir de dispersion des colonisateurs.

Figure 1. Représentation graphique de la théorie des cycles de taxons

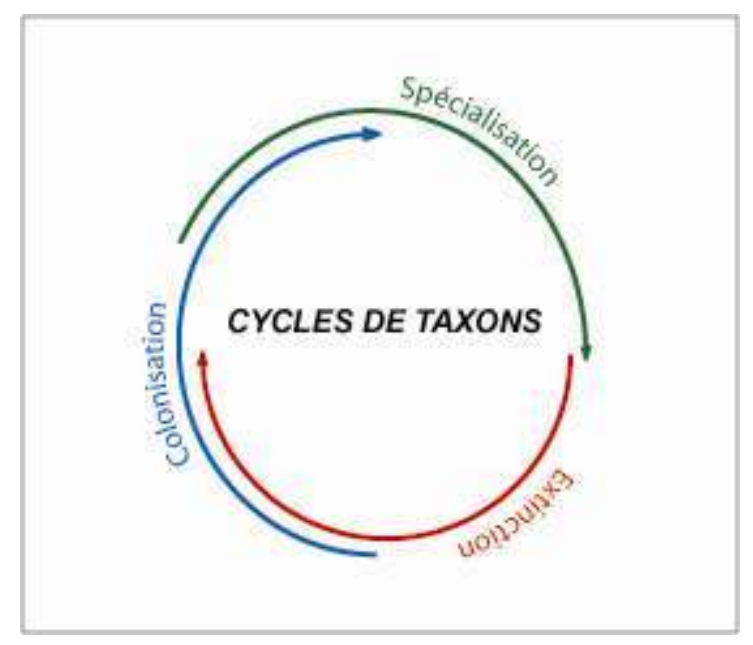

Source : auteur (2011)

9 Aujourd'hui, à cause d'une diminution accrue de l'isolement, l'anthropisation amplifie de beaucoup la vulnérabilité des îles. En effet, depuis la découverte de nouvelles technologies et l'accélération du savoir, l'insularité, telle qu'elle est perçue pour les îles océaniques, semble compromise. En facilitant leur accessibilité, l'amélioration des moyens de transport a d'abord favorisé des modifications dans la composition et la structuration de l'espace. Plus encore, l'accroissement des échanges entre les continents et les îles a permis de transporter régulièrement d'importantes quantités d'organismes exotiques. Les ports de la Réunion, de la Martinique, de la Guadeloupe et de Madagascar affichent un trafic de conteneurs aussi important que des ports continentaux (Nicolas, 2005). Ainsi replacés dans des niches écologiques vacantes, certains de ces taxa, devenus invasifs, purent s'installer et se développer en toute quiétude au détriment d'autres taxa endémiques, mettant ainsi en danger la diversité biologique originelle des espaces insulaires. Les perturbations d'origine anthropique ont, en effet, tendance à générer des 
conditions favorables à des espèces rudérales ou pionnières, tout en limitant la survie de taxa propres au milieu ou de fin de succession : leurs effets sur la présence d'espèces rares ou endémiques seraient souvent négatifs (Lepart, 2005). Christophe Grenier propose la notion de continentalisation des milieux insulaires pour caractériser l'ouverture, l'intégration et la transformation des îles dans le système monde (Grenier, 1996). Sa thèse sur la conservation, le tourisme et les migrations aux îles Galápagos, démontre que les réseaux de transport et de communication ont permis de réduire l'espace/temps terrestre engendrant dès lors l'érosion de la biodiversité. En effet, la densification progressive des réseaux d'usagers - touristes et migrants principalement - aux Galápagos a modifié l'espace d'évolution en mettant en communication rapide et régulière des étendues et des espèces anciennement séparées les unes des autres. Ainsi, des taxa continentaux à tendance généraliste (opportunistes, à courtes générations, à grand pouvoir de dispersion, capables de s'adapter rapidement à une grande variété de biotopes) mettent en péril un certain nombre d'espèces endémiques aux Galápagos qui, pour certaines, sont menacées de disparition à court terme. En ouvrant son analyse à l'île de Pâques, il en arrive à la conclusion que le rattachement de ces deux espaces insulaires à l'Amérique a eu pour conséquence une destruction effrénée de leur nature qui, en s'étendant aux autres écosystèmes insulaires, entraînera à terme la diminution du nombre des lieux permettant la spéciation géographique et, par conséquent, la réduction de la diversité terrestre (Grenier, 2002). Ce processus d'anthropisation se traduit donc par diverses menaces dont les conséquences sur la diversité biologique ont largement été démontrées (Whittaker, 1998). Par exemple, le nombre d'oiseaux, de mollusques, de mammifères, de reptiles, d'amphibiens et d'insectes éteints depuis 1600 varierait entre 94 et 97 pour des espaces continentaux tandis qu'il serait estimé à près de 355 pour des régions insulaires.

Tableau 2. Extinction de quelques taxa insulaires et continentaux selon Groombridge (1992) et Steadman (1997)

\begin{tabular}{|l|l|l|}
\hline Classes & $\begin{array}{l}\text { Nombre d'espèces éteintes dans des } \\
\text { régions insulaires }\end{array}$ & $\begin{array}{l}\text { Nombre d'espèces éteintes dans des } \\
\text { régions continentales }\end{array}$ \\
\hline Oiseaux & 97 & 17 à 20 \\
\hline Mollusques & 151 & 40 \\
\hline Mammifères & 34 & 24 \\
\hline Reptiles & 22 & 1 \\
\hline Amphibiens & - & 2 \\
\hline Insectes & 51 & 10 \\
\hline
\end{tabular}

Sources : Whittaker (1998)

10 Cela conduit actuellement à l'une des problématiques majeures mises en évidence à la fin $\mathrm{du} \mathrm{XX}^{\mathrm{e}}$ siècle: les hauts lieux de la diversité biologique. Les hotspots sont des régions bénéficiant d'une extraordinaire concentration d'espèces et subissant une perte accélérée d'habitats. Ces points chauds menacés de disparition, ou zones critiques de conservation, sont répartis sur l'ensemble du globe. Leur richesse en espèces de plantes vasculaires et 
en vertébrés terrestres, et plus particulièrement en endémiques, a été largement démontrée (Myers et al., 2000 ; Mittermeier et al., 2005). L'idée de proposer des régions prioritaires a pour objet de guider la politique de conservation de la biodiversité en délimitant des unités spatiales où il est nécessaire de concentrer les investissements afin de limiter efficacement l'extinction mondiale des espèces. Les hotspots concentrent sur $1,4 \%$ de la surface terrestre $44 \%$ du nombre total d'espèces de plantes vasculaires et $35 \%$ de toutes les espèces de quatre groupes de vertébrés (oiseaux, mammifères, reptiles et amphibiens). Myers (2000) a proposé 25 régions constituant les points chauds de la diversité biologique. Mittermeier (2005) complète ce chiffre par 9 autres zones critiques de conservation de la diversité biologique. Les îles occupent une place particulière au sein des zones critiques de conservation de la diversité biologique. Ces hauts lieux comptent huit régions insulaires : les îles polynésiennes et micronésiennes, la Caraïbe, Madagascar, les Philippines, les îles de la Sonde, la Nouvelle-Zélande, la Nouvelle-Calédonie et les îles regroupées en région de Wallace. L'attention qui semble se porter sur les espaces insulaires pour optimiser la conservation de la diversité biologique s'explique par leur degré d'endémisme. Ces espaces regroupent 35,1\% des 133149 espèces de plantes vasculaires endémiques et $38,8 \%$ des 9645 espèces de vertébrés terrestres recensées dans l'ensemble des hotspots. Madagascar, les îles de la Sonde et la Caraïbe insulaire sont parmi les cinq hotspots où le nombre d'espèces endémiques est le plus important (Myers et al., 2000). Ce sont des espaces prioritaires pour l'étude des problématiques de la diversité biologique et plus largement de la biodiversité interprétée comme une idéologie notamment par les sciences sociales.

\section{La biodiversité, du néologisme à l'idéologie}

11 La problématique de la biodiversité a été motivée par l'inquiétant constat d'une accélération d'origine anthropique du phénomène d'extinction biologique. Utilisé par Thomas Lovejoy en 1980 au sein de l'UICN, biological diversity a été simplifié par le néologisme biodiversité lors de la publication du premier compte-rendu du forum américain sur la diversité biologique, organisé en 1986 par le conseil scientifique national (National research council). La publication du livre d'Edgar O. Wilson, BioDiversity (1988), marquera l'émergence du concept. Il est issu de la prise de conscience de l'impact de l'homme sur les milieux naturels et sur leurs ressources biologiques. Longtemps abordées par les sciences naturelles, ces problématiques seront considérablement étendues aux sciences humaines et sociales à partir de 1992, suite à la Conférence de Rio sur l'environnement et le développement durable qui précéda la ratification de la Convention sur la diversité biologique en 1993. Le sommet mondial de Johannesburg sur le développement durable a plus que jamais relancé le débat autour de cette problématique mondiale. Plus récemment, le sommet de Nagoya le 29 octobre 2010, renforce l'attention portée aux problématiques de biodiversité. Cet intérêt pour la diversité biologique - sa préservation, la préconisation d'usages durables, le partage équitable - a fait l'objet de nombreuses réflexions abordant la biodiversité à partir de la biologie, de la géographie, de la sociologie, de la psychologie, de l'économie, du droit et de la politique (Marty et al., 2005). L'analyse de ces différentes approches de la biodiversité amène à distinguer son approche traditionnellement biocénotique où la science fait état de la diversité du vivant, d'une perception, plus novatrice, dans laquelle le concept engloberait aussi sa 
préservation, d'en préconiser des usages durables, de veiller à un partage équitable des bénéfices qu'elle apporte (Barbault et al., 2005).

En 1987, le bureau d'évaluation technologique du congrès américain, Office of technology assessment, postula que la diversité biologique se rapporte à la variabilité et à la variété parmi les diverses formes de vie et dans les complexes écologiques dans lesquels elles se rencontrent . McNeely étaye cette définition en citant les groupes d'êtres vivants et les complexes écologiques, soit l'ensemble des espèces de plantes, d'animaux et de micro-organismes ainsi que les écosystèmes et les processus écologiques. Dès lors, la diversité biologique représentera le degré de variété naturelle incluant à la fois le nombre et la fréquence des écosystèmes, des espèces et des gènes dans un ensemble donné ${ }^{5}$. En effet, les systèmes biologiques sont des ensembles complexes, hétérogènes et hiérarchiquement emboîtés. Les atomes s'agencent en cristaux ou en molécules qui s'organisent en cellules capables de se reproduire. L'agrégation et la coopération entre les cellules constituent des organismes multicellulaires. L'individu, unicellulaire ou pluricellulaire ainsi constitué, est considéré comme l'unité élémentaire du monde vivant. Il est porteur d'un patrimoine génétique propre qui constitue son génotype et dont l'expression sera le phénotype. Les individus susceptibles d'échanges génétiques fertiles et féconds sont regroupés en espèces. Les individus d'une même espèce habitant un même milieu s'organisent en populations. Les ensembles plurispécifiques délimités généralement à partir des bases de la taxinomie forment des peuplements ou des communautés. L'ensemble des populations d'espèces animales et végétales qui évoluent au sein d'un milieu donné constitue une biocénose. En définissant la diversité biologique par la variété de ses composantes à différentes échelles d'analyse, les précédents auteurs semblent minimiser son aspect fonctionnel. Sandlund (1993) comblera cette lacune en attribuant à la diversité biologique la variété fonctionnelle des diverses formes de vie qui peuplent la biosphère aux niveaux d'organisation et de complexité croissante: génétique, population, espèce, communauté, écosystèmes. Jacques Blondel (1995) aborda son aspect pratique en la caractérisant par la quantité et la structure de l'information contenue dans des systèmes vivants hiérarchiquement emboîtés. Elle s'appréhende donc selon différents niveaux interdépendants et hiérarchiquement emboîtés. On parlera de diversité génétique intra et inter-populations, de diversité spécifique, de diversité des assemblages d'espèces, de diversité des écosystèmes au sein des paysages et de diversité dans le temps de systèmes biologiques changeants qui évoluent. De manière théorique, l'expression diversité biologique désigne donc un ensemble d'entités d'importance et de complexité variables et croissantes (Ramade, 2002).

13 Les propositions précédemment exposées laissent prétendre que ce concept de diversité biologique est utilisé pour déterminer le degré de variabilité dans la complexité de systèmes biologiques structurés dans lesquels chaque élément est aussi caractérisé par des fonctions. Ces systèmes sont hiérarchiquement emboîtés selon des niveaux d'organisation des échelles biosystémiques intracellulaire, cellulaire et organique (Lacoste et al., 2001). Cela signifie que l'étude de la diversité biologique peut être approchée à différentes échelles de complexité et ce en fonction des critères élémentaires, structuraux et fonctionnels. L'approche biologique aborde donc la diversité biologique selon trois niveaux de perception: la diversité génétique, la diversité taxinomique et la diversité écosystémique qualifiées respectivement de diversité génétique, organistique et écologique (Blondel, 2005; Simon, 2007). Bien que l'approche génétique ait une importance fondamentale dans la connaissance des unités élémentaires de la diversité biologique, la notion s'identifie plus fréquemment aux espèces. À l'échelle taxinomique, la 
diversité biologique se réfère aux activités concernant l'inventaire et la connaissance du monde vivant. Enfin, la diversité des écosystèmes, qualifiée d'écodiversité (Blondel, 2005) ou de biocomplexité (Lévêque et al., 2001), constitue les interactions fonctionnelles entre les entités biologiques.

Dans son acception originelle, le néologisme biodiversité est utilisé comme synonyme de l'expression diversité biologique. Les modalités d'utilisation actuelle du mot tendent à lui apporter une autre dimension. Le dictionnaire de l'environnement dirigé par Yvette Veyret (2007), aborde la notion de biodiversité en se référant à sa triple dimension biologique, politique et juridique. En effet, si pour certains la biodiversité n'est qu'une manière d'aborder la complexité du vivant sous toutes ses formes et à toutes ses échelles spatiales, pour d'autres disciplines sa diffusion et son appropriation lui confèrent une signification plus large. Elle fait partie des enjeux majeurs du vingt et unième siècle. En effet, dans l'esprit de la Convention sur la diversité biologique et de ses pays signataires, la notion de biodiversité ne se limite pas uniquement à inventorier le vivant (Barbault et al., 2005; Veyret, 2007). Sur des prétextes variés faisant souvent appel à des considérations éthiques, esthétiques, culturelles et économiques, le terme biodiversité est devenu une véritable idéologie à laquelle les politiques gouvernementales acceptent ou non d'adhérer. Ce concept s'utilisera pour parler des questions relatives aux interactions entre les hommes et la nature. Il s'applique à tout ce qui relève de l'érosion du monde vivant résultant des activités humaines, aux activités de protection et de conservation, à la création d'espaces naturels protégés et aux modifications de comportements en matière de développement. Cette approche élargie du concept fait référence à des problématiques telles les modes d'accès à la diversité biologique, les usages qu'en font les sociétés, les savoirs développés pour y accéder, les bénéfices qu'on en retire, la manière dont ces bénéfices doivent être partagés, sa gestion et sa durabilité (Blondel, 2005; Boisvert et al., 2005). Dans ce registre institutionnel la biodiversité se réfère aux différentes actions menées en faveur de la diversité biologique.

Dans cette perception modernisée où la relation de l'homme, et plus globalement des sociétés, à la nature est l'élément fondateur, la biodiversité est utilisée pour parler de préoccupations variées, selon que l'on se place du point de vue de la systématique, de l'économie, du droit, de la sociologie, de la psychologie ou de la géographie (cf. figure 2). Le systématicien et plus généralement le naturaliste abordent la biodiversité comme la diversité de toutes les formes du vivant (Lepart, 2005). L'économiste ou l'ingénieur s'attachera à tout ce qui concerne son exploitation et sa valorisation (Vivien, 2005). Le juriste et le politologue privilégieront l'élaboration, la discussion et le respect des textes visant à institutionnaliser le concept (Maljean-Dubois, 2005). Le sociologue et l'historien veilleront à étudier l'organisation des sociétés humaines autour de la biodiversité (Micoud, 2000 ; Fortier et al., 2005 ; Selmi, 2005). 
Figure 2. Biodiversité à la croisée des systèmes

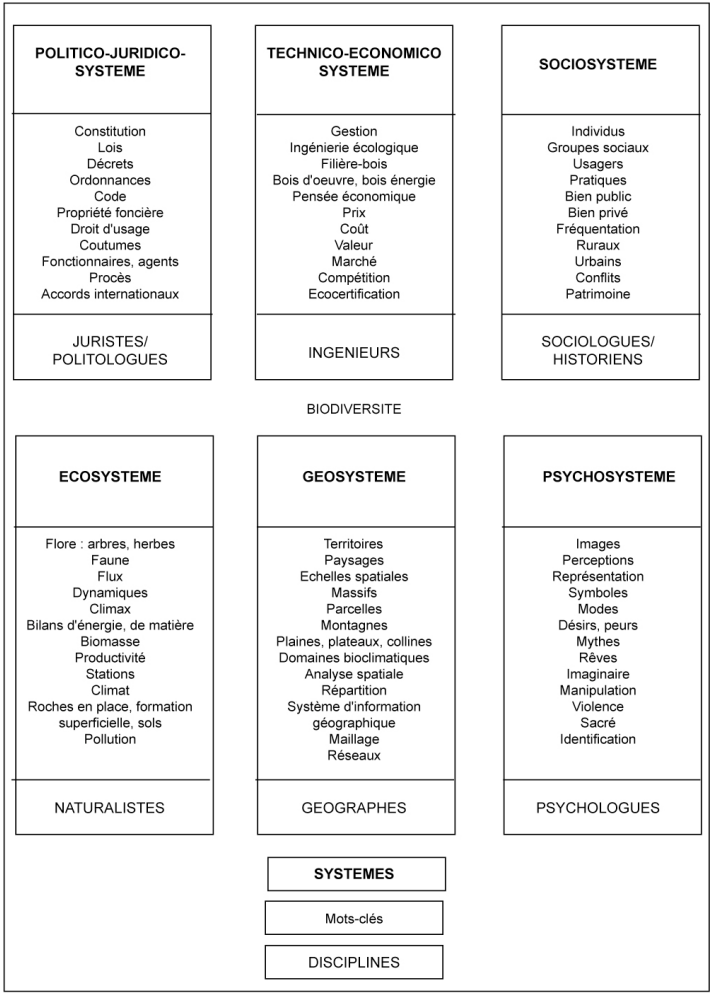

Source : Arnould (2005)

Malgré leurs différences, toutes ces visions de la biodiversité ont la même finalité : la conservation des différentes expressions de la diversité biologique. Comme l'a clairement souligné Jacques Blondel (2005), la biodiversité n'est pas une discipline de recherche, mais une nouvelle manière d'aborder des champs de recherche traditionnels comme la biogéographie, l'écologie, la systématique et la génétique pour ce qui est des sciences de la nature. Elle s'intéresse aux acteurs que sont les entités biologiques le long des hiérarchies biologiques, des gènes aux paysages, mais aussi à leurs fonctions sociales et aux services qu'ils rendent. Ainsi, le mot biodiversité s'emploiera pour parler de l'ensemble des interactions entre la diversité biologique et son environnement. L'expression diversité biologique sera utilisée pour décrire la variabilité du vivant. Elle s'opère différemment selon les niveaux d'organisation ou d'intégration des échelles biologiques et spatiales. La diversité biologique et plus généralement la biodiversité ne peuvent s'envisager dans leur globalité ; il nous a donc semblé nécessaire d'illustrer cette réflexion en analysant la biodiversité à la Martinique au travers de l'une de ces composantes biologiques, l'avifaune.

\section{L'exemple d'une île française dans la Caraïbe, des initiatives de gestion en faveur de la biodiversité avienne à la Martinique}

Dans la Caraïbe, le territoire de la Martinique est une illustration du positionnement politique français dans la gestion des hauts lieux de la diversité biologique mondiale. Considérant les deux unités insulaires de la Basse-Terre et de la Grande-Terre pour 
l'archipel de la Guadeloupe (Westercamp et al., 1980), ce département français est l'île la plus vaste de l'archipel des Petites Antilles. D'une superficie d'environ $1100 \mathrm{~km}^{2}$, la Martinique s'étend du nord-ouest au sud-est sur une longueur de $73 \mathrm{~km}$. Sa forme très allongée lui confère une largeur maximale de $39 \mathrm{~km}$. Elle se situe entre le tropique du cancer et l'équateur au $14,5^{\circ}$ de latitude nord et $61^{\circ}$ de longitude ouest (cf. figure 3). Elle est baignée à l'ouest par la mer des Caraïbes et à l'est par l'océan Atlantique. Elle compte près d'une dizaine d'espèces de reptiles et de mammifères, contre plus d'une soixantaine d'espèces d'oiseaux nicheurs. Bien que les diversités en espèces aviennes de la France métropolitaine (276) et de la Guyane (718) soient largement supérieures à celle de la Martinique, le taux d'endémisme (3\%) y est plus élevé. Plus encore, sur moins de $1 \%$ de la superficie de la France métropolitaine associée aux autres départements d'outre mer, on dénombre plus de $1 / 10^{\text {ième }}$ de sa richesse en espèces d'oiseaux. On estime à près de 200 le nombre d'espèces d'oiseaux fréquentant la Martinique. Bird life international a identifié la Martinique comme étant une Zone d'endémisme pour les oiseaux des Petites Antilles.

Figure 3. La Martinique dans l'archipel des Petites Antilles

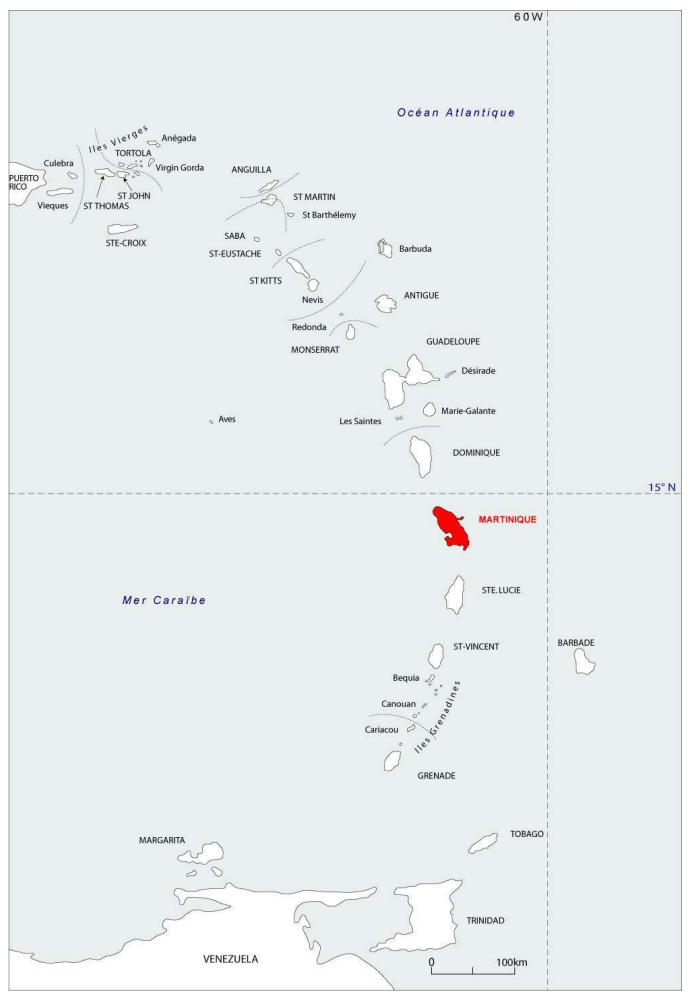

Source : Géode Caraïbe (2005)

Les modalités de gestion durable et intégrée auxquelles sont soumis des espaces insulaires, peuvent, à certains égards, s'apparenter à une forme de commensalisme voire de mutualisme. En effet, l'homme s'est intéressé à la protection des oiseaux pour des raisons éthiques : conscient de sa responsabilité dans la raréfaction et l'extinction de certaines espèces, on peut comprendre que des considérations morales l'aient poussé à se racheter auprès d'une nature dont dépend sa survie, mais plus encore qu'il accepte de partager avec d'autres espèces. À la Martinique, les sites les plus représentatifs de cette démarche sont les îlets ${ }^{6}$ de la presqu'île de Sainte-Anne. Le décret de mise en réserve naturelle de ces îlots a pour objectif prioritaire de protéger les espèces pélagiques qui s'y 
reproduisent. Cet espace dédié aux oiseaux se compose des îlets Hardy, Poirier, Burgeaux et Percé (cf. figure 4). Ils totalisent une superficie de 5,76 hectares dont 2,63 hectares pour l'îlet Hardy, 2,1 hectares pour l'îlet Poirier, 0,49 hectares pour l'îlet Burgeaux et 0,54 hectares pour l'îlet Percée. Robert Pinchon signalait déjà l'importance de ces îlets pour les colonies d'oiseaux qui viennent y établir leurs nids chaque année (Pinchon, 1976). Les sternes (Sterna fuscata, Sterna anaethetus, Sterna dougallii, Sterna hirundo) et le Noddi niais ( Anous stolidus) pondent généralement leurs œufs à même le sol sur les tapis herbacés que forment le Pourpier-bord-de-mer (Sesuvium portulacastrum) et l'Herbe-bord-de-mer ( Sporobolus virginicus), ou dans les anfractuosités des îlots, et le réseau de galeries calcaires de l'îlet Hardy pour le Puffin d'audubon (Puffinus lherminieri). D'autres pélagiques d'intérêt, tel le Paille-en-queue (Phaethon aethereus), nichent aussi dans la réserve. Des limicoles, tel le Tournepierre à collier (Arenaria interpres), fréquentent les parties sableuses de l'îlet Hardy. Cette démarche préservationniste ne fait l'objet d'aucune forme de valorisation. Au contraire, la visite des îlets est strictement interdite au public.

Figure 4. Les îlets de la réserve naturelle ornithologique à la Martinique

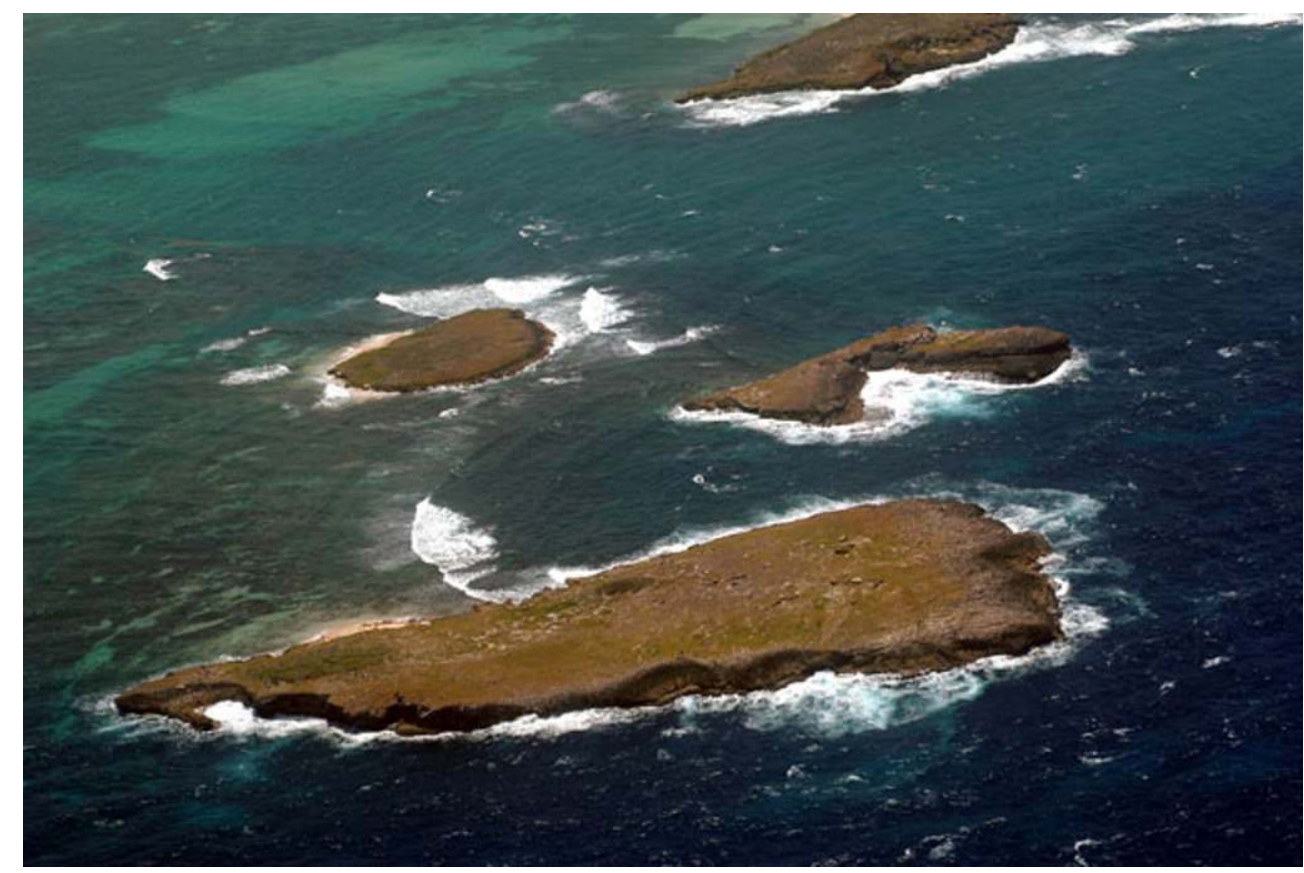

Source : cliché Parc naturel régional de la Martinique (2010)

En outre, en attribuant aux oiseaux une fonction de ressource biologique au travers d'activités ludiques, la démarche conservationniste à leur égard est d'autant plus justifiée par rapport aux avantages que la société en tire dans la perspective de développer l'écotourisme ornithologique. Actuellement, les aspects ludiques de l'ornithologie à la Martinique sont principalement motivés par des associations de naturalistes. Leurs activités peuvent être classées en plusieurs regroupements : les actions d'amélioration de la connaissance scientifique et de suivi des espèces ; les actions de sauvegarde ; les actions de sensibilisation du grand public aux problématiques de conservation et de protection des oiseaux à la Martinique; et les actions de formation à l'ornithologie. L'une des principales sources de revenus des îles de la Caraïbe, le tourisme, n'est que très partiellement incluse dans leurs activités. L'observation de spécificités ornithologiques, véritable source de profits dans certaines régions du monde, ne fait pas partie des attraits 
mis en avant dans la politique de médiatisation touristique à la Martinique. Or, l'observation d'oiseaux pour le plaisir, plus communément appelée birding ou birdwatching chez les anglo-saxons, est une activité en plein essor. Céline Chadenas (2003) utilise l'expression d'internationalisation de l'observation ornithologique lorsqu'elle aborde l'engouement mondial pour cette forme d'écotourisme. Ce secteur en plein développement est perçu à certains égards comme étant une chance pour la prise de conscience environnementale de chacun, mais également une opportunité économique.

L'avifaune tend à être perçue comme l'une des valeurs les plus sûres de l'écotourisme martiniquais, sur laquelle il devient primordial de mettre l'accent par le biais d'aménagements spécifiques. C'est à partir de 2004 que l'étang des Salines, déjà apprécié par les chasseurs pour sa richesse ornithologique en espèces migratrices, fit l'objet d'aménagements en vue de réaliser un parcours découverte. Afin de faire découvrir cet étang et les biotopes auxquels il est associé, le Conservatoire des espaces littoraux et de rivages lacustres a construit une structure en bois permettant l'observation de la flore et de la faune, sans pour autant nuire à la tranquillité des espèces présentes sur ce site. L'outil de tourisme ornithologique, réalisé en 2006, est un parcours qui se prolonge en une véritable promenade au-dessus de l'étang avec au milieu une palissade d'observation de la faune (cf. figure 5). Il se présente comme une fenêtre ouverte sur un paysage aquatique permettant une meilleure observation des oiseaux migrateurs qui fréquentent les vasières de l'étang. Bien que l'objectif de tourisme ornithologique ne soit qu'un des aspects mis en avant dans la valorisation du site, il est perçu comme l'un des aménagements qui marque les prémices d'un birdwatching dans le département de la Martinique.

Figure 5. Observatoire de la faune et de la flore des étangs à la Martinique
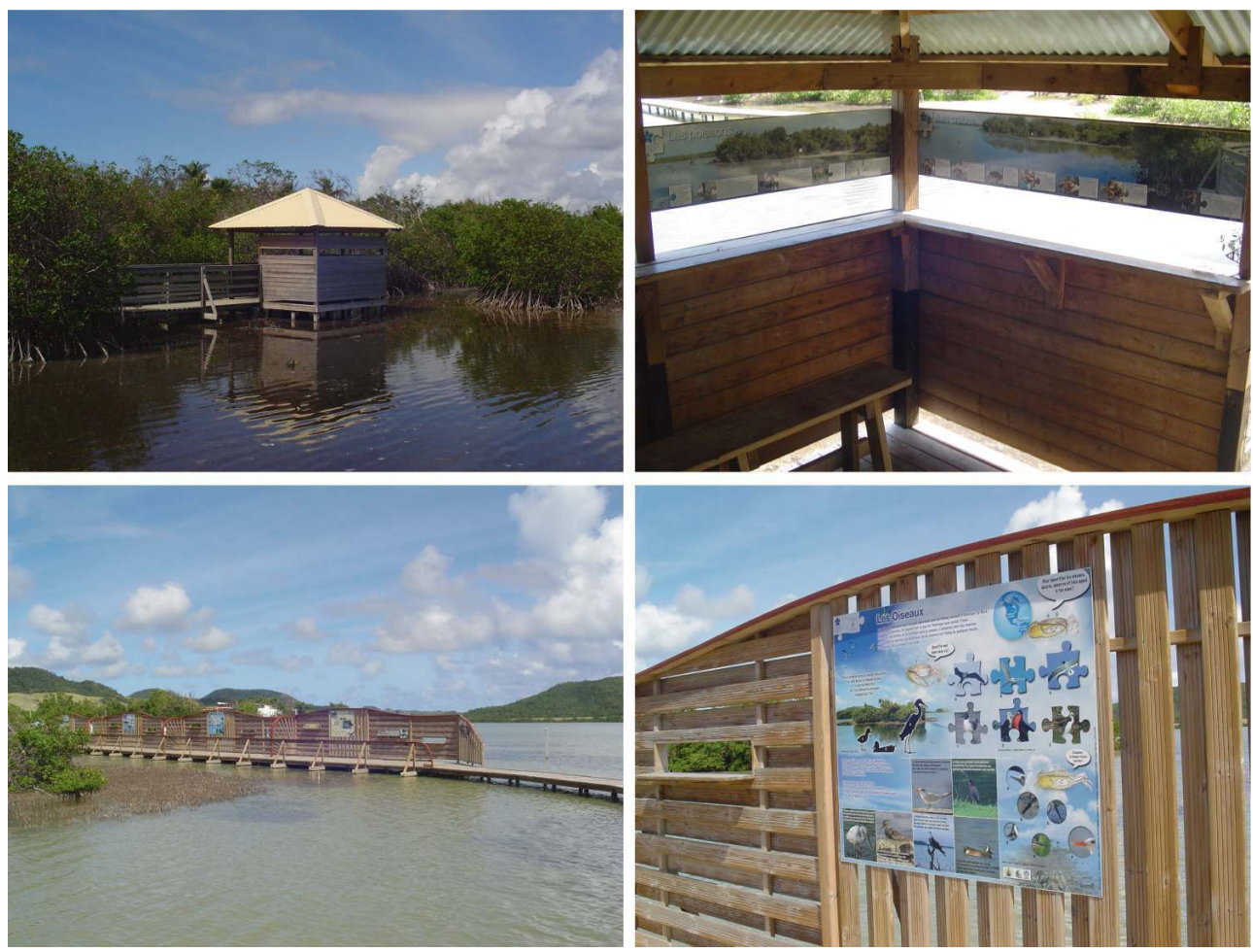

Source : auteur (2007) 
Dans cet objectif de valorisation de l'avifaune et des espaces associés, soit des entités biospatiales ${ }^{7}$, le Conservatoire du littoral et des rivages lacustres a installé un système de vidéotransmission de la faune aviaire sur l'îlot du Rocher du Diamant. L'objectif principal de cette technologie est de faire découvrir en temps réel à un large public les populations d'oiseaux qui fréquentent cet îlot. En effet, pas moins de treize espèces ont été observées en 2004. Parmi celles-ci, le Noddi brun (Anous stolidus), la Sterne bridée (Sterna anaethetus), le Paille en queue (Phaethon aethereus), le Fou brun (Sula leucogaster) et la Frégate superbe ( Fregata magnifiscens). De récentes observations citent même la présence du Puffin d'audubon (Puffinus lherminieri), une espèce pour laquelle les enjeux de conservation sont prioritaires à l'échelle internationale. Le Her Majesty's Diamond Rock, comme le baptisèrent les Anglais en 1805, est protégé par un Arrêté de protection de biotope depuis 1994. L'accès y est actuellement interdit en raison de la présence de plus d'un millier d'oiseaux marins qui s'y arrêtent chaque année. Il constitue un attrait touristique majeur. Cependant, il n'existait aucun aménagement favorable à une valorisation pédagogique et plus encore écotouristique de l'îlot. L'outil de valorisation consiste à filmer le comportement des oiseaux nichant ou fréquentant le Rocher à partir de caméras amovibles, disposées à des zones de concentration d'oiseaux. Un pupitre de télécommande des caméras contrôle leurs prises de vue. Il est également prévu la mise à disposition d'un ordinateur qui fera la liaison avec internet, ainsi que l'enregistrement des meilleures séquences pour une présentation aux visiteurs. Les internautes pourront ainsi avoir des images du rocher en tous temps.

Ces exemples appuient le postulat énoncé par Claude Raffestin en 1980 : tout objet peut endosser une fonction patrimoniale et tout espace peut devenir territoire à condition qu'ils soient l'un et l'autre, pris dans un rapport social de communication (Di Méo, 2001). Or, dans un contexte régional où le maintien et la création d'emplois sont des objectifs prioritaires, la construction d'objets patrimoniaux biospatiaux pourrait appuyer le développement économique. Plus encore, en devenant des actifs, ces ressources spécifiques (Colletis et al., 2005) feraient office de signaux qui attireraient des consommateurs, notamment des touristes, qui apprécieraient aussi la qualité des autres produits d'un panier de biens et services territorialisés (Pecqueur, 2002). Formalisée par Bernard Pecqueur en 2001, l'hypothèse du panier s'appuie sur l'idée que : à l'occasion de l'acquisition d'un produit de qualité territoriale, le consommateur découvre la spécificité des autres produits issus de la production locale et détermine son utilité sur l'ensemble des produits offerts. L'objet patrimonial peut dès lors être construit comme l'une des composantes attractives d'une offre composite - notamment touristique - pensée à l'échelle de l'ensemble du territoire (François et al., 2006 ; Landel et al., 2009).

La Réserve naturelle de la Presqu'île de la Caravelle, actuellement gérée par le Parc naturel régional de la Martinique, est un territoire propice pour expérimenter le passage du statut de ressource à celui d'actif spécifique pour un objet patrimonial dans une logique de panier. Cet espace protégé fut créé, en 1976, dans le but de valoriser le patrimoine géologique naturel et paysager de l'une des plus anciennes parties émergées de la Martinique, de préserver et de conserver la diversité biologique du milieu tropical sec caractéristique des Petites Antilles, ainsi que de protéger le biotope de nombreuses espèces d'oiseaux (Edouard et al., 1995). L'avifaune qui se développe sur cette extrémité de petite péninsule comprend une grande variété d'espèces dont les plus remarquables sont deux oiseaux prioritaires en matière de conservation, le Moqueur gorge blanche ( Ramphocinclus brachyurus) et l'Oriole de Martinique (Icterus bonana). Sa seconde fonction 
est d'entretenir et de valoriser un lithôme touristique (Defert, 1982; Lozato-Giotart, 2003): le monument historique des ruines du château Dubuc. Le parc naturel régional estime que sa fréquentation annuelle varierait entre trente et quarante milles visiteurs essentiellement constitués de scolaires et de touristes. Il est classé parmi les trois musées les plus visités (Bégot, 2009). La valorisation de ce patrimoine historique s'appuie principalement sur les légendes racontées autour des mystères de la famille Dubuc. Les entretiens réalisés auprès des quatre professionnels du Parc naturel régional de la Martinique chargés des visites touristiques autorisent une probable distinction des touristes selon leur motivation : ceux qui sont strictement attirés par les ruines du château Dubuc ne sont généralement pas motivés par la découverte immédiate des paysages, de la flore et de la faune de la réserve. Or, l'offre initialement proposée par l'office du tourisme, suggère d'accompagner la visite des ruines par une marche sur les sentiers de la réserve. En outre, ceux qui terminent un itinéraire sont dans de meilleures dispositions pour découvrir ce site historique. Ce constat a notamment été vérifié lors d'une expérience de birding à la Réserve naturelle de la Caravelle en 2010. Bien que l'unique motif de la visite d'un groupe d'une quarantaine d'ornithologues, majoritairement originaires des ÉtatsUnis, était d'enregistrer des chants d'oiseaux et de photographier le Moqueur gorge blanche et l'Oriole de Martinique, ils ont manifesté un intérêt apparent pour la visite des ruines et les présentations sur l'histoire de la famille Dubuc. Ce constat conforte l'existence d'une perspective de panier de biens et services territorialisés applicable à la Réserve naturelle de la Caravelle: à l'occasion d'une expérience d'observation ornithologique et plus largement de découverte d'un patrimoine naturel, le touriste consommerait aussi un produit patrimonial historique. Par son attractivité avérée, le patrimoine historique pourrait aussi être ce signal dont la principale fonction serait de conduire le touriste à découvrir et à apprécier les spécificités d'un autre produit du panier de biens et services territorialisés (Pecqueur, 2002).

24 Afin de parvenir à la construction d'un produit touristique, il est nécessaire que cet objet patrimonial passe de l'état de ressource spécifique à celui d'actif spécifique. Actuellement, ce changement de fonction est probablement limité par des stratégies de valorisation qui sont spécifiques à chacun des objets patrimoniaux. Bien qu'ils appartiennent tous les deux au territoire de la Réserve naturelle de la Caravelle, il n'existe pas de produit touristique conçu de manière à lier la découverte des particularités géologiques, paysagères, floristiques et faunistiques, à la visite du site historique des ruines du château Dubuc. Si la présence d'un personnel commun à l'animation et à la gestion des deux objets pose les bases d'une valorisation commune, la construction du produit touristique ne peut s'envisager sans une intégration d'acteurs privés dans le plan de gestion de la réserve. Une telle initiative risquerait d'être confrontée aux difficultés de concilier les objectifs des secteurs publics et privés. Dans une logique publique, le patrimoine est un bien collectif dont la valorisation privée conduirait à une appropriation individuelle. Les travaux de Bernard Pecqueur (2002) suggèrent de dépasser ce dilemme privé-public en appliquant une logique mixte dans laquelle les acteurs valoriseraient le processus de patrimonialisation bien plus que les sites eux mêmes.

Il nous semble donc nécessaire que, par-delà la conservation, la diversité biologique devra s'affirmer au travers d'une valeur capable de justifier économiquement les démarches de gestion durable et intégrée entreprises à son égard. Face aux besoins fonciers nécessaires au développement local, l'élaboration d'une fonction économique de l'avifaune serait 
probablement un argument particulièrement pertinent qui justifierait plus concrètement le choix de conservation de milieux naturels auprès des Martiniquais. En percevant l'oiseau comme l'un des objets patrimoniaux d'un panier de biens et services territorialisés, la conservation des entités biospatiales, par la mise en protection de certaines zones, serait autrement appréhendée par les autochtones.

\section{Conclusion}

Une approche biocentriste (Larrère, 2005) serait utopique dans une biosphère dont l'orientation et le foisonnement de la diversité biologique sont principalement liés aux processus de compétition interspécifique (Lamy, 1999). Les changements spatiotemporels de composition et de structuration de la diversité biologique sont inexorablement dépendants du phénomène d'exclusion compétitive. Il est donc normal que, pour assurer sa survie, l'espèce humaine rentre en compétition avec d'autres taxa dans l'exploitation de l'espace et de ses ressources. La lutte pour l'existence est un phénomène normal où les taxa les mieux adaptés à un environnement s'imposent face aux moins biens adaptés. Rappelons par exemple que l'accroissement des effectifs d'espèces aviennes partageant les mêmes ressources alimentaires et les mêmes lieux de reproduction de la Bernache cravant (Branta bernicla) est à l'origine de la diminution de leur population en Europe (Chadenas, 2008). En tant qu'espèce, l'homme répond parfaitement aux règles qui régissent la biosphère. Néanmoins, pour diverses raisons (éthiques, esthétiques, économiques, sociologiques, culturelles, écologiques), sa sphère intellectuelle ou noosphère lui impose de prendre en considération la conservation de la vie sous toutes ses formes. Il a dès lors la responsabilité de pérenniser sa propre existence dans un altruisme tourné vers les besoins des générations futures, mais aussi les besoins d'autres entités biologiques avec lesquelles il partage la biosphère.

Une démarche écocentriste, intégrant des initiatives de gestion favorables à la biodiversité dans son acception idéologique, est un objectif prioritaire de développement dans les territoires insulaires. La gestion future des petites îles devra permettre de corriger les effets pervers du développement, par la définition d'actions modèles, applicables s'il le faut dans d'autres parties du monde, et basées sur la qualité de la vie et sur la durabilité, beaucoup plus que sur une croissance en soi (Burac, 2006). Les raisons et les choix de préservation doivent dès lors être fixés selon les lieux et les circonstances. L'enjeu de la conservation de la biodiversité est en réalité celui du développement durable (Boutaud, 2004) et du bien-être des nations (OCDE, 2001 ; Prescott-Allen, 2003) en recherchant prioritairement la viabilité des systèmes de gouvernance dans les pays du Sud, la capacité de l'État central à faire respecter des règles générales fixées par les régimes internationaux, tout en reconnaissant aux communautés locales un véritable pouvoir sur la ressource (Compagnon, 2008). Les modalités de conservation et de valorisation d'une entité biospatiale doivent dépendre du contexte social, culturel et économique, analysé à une échelle de gestion territoriale locale replacée dans un cadre international. 


\section{BIBLIOGRAPHIE}

Arnould P., 2005, « Biodiversité : quelle histoire? », in P. Marty and al. (eds.), Les biodiversités, Paris, CNRS éditions, p. 67-80.

Barbault R, Chevassus-au-Louis B., 2005, Biodiversité, science et gouvernance, Paris, ADPF, 244 p.

Bégot D., 2009, «Musées et politique patrimoniale des DOM antillais : l'exemple de la

Martinique ", in J.M. Breton (eds.), Patrimoine culturel et tourisme alternatif, Paris, éditions

Karthala, p. 107-123.

Blondel J., 2005, « Biodiversité et sciences de la nature », in P. Marty and al. (eds.), Les biodiversités , Paris, CNRS éditions, p. 23-36.

Blondel J, 1995, Biogéographie, Paris, Masson, 297 p.

Blondel J., 1986, Biogéographie évolutive, Paris, Masson, 218 p.

Blondel J., 1979, Biogéographie et écologie, Paris, Masson, 172 p.

Boisvert V., Vivien F.D., 2005, « Tiers Monde et biodiversité : tristes tropiques ou tropiques d'abondance ? La régulation internationale des ressources génétiques mise en perspective », Tiers-Monde, vol. 46, n 181, p. 185-206.

Boutaud A., 2004, Le développement durable : penser le changement ou changer le pansement?, SaintÉtienne, École Nationale Supérieure des Mines, 415 p.

Burac M., 2006, «Quel développement dans les petites îles tropicales ?», in R. Confiant and al. (éds.), À l'arpenteur inspiré, Matoury, Ibis rouge, p. 371-398.

Chadenas C., 2008, L'Homme et l'Oiseau sur les littoraux d'Europe occidentale, Paris, L'Harmattan, $293 \mathrm{p}$.

Chadenas C., 2003, L'Homme et l'oiseau sur les littoraux d'Europe occidentale (appropriation de l'espace et enjeux territoriaux : vers une gestion durable ?), Nantes, Université de Nantes, 242 p.

Colletis G., Pecqueur B., 2005, « Révélation de ressources spécifiques et coordination situéeé, in D. Talbot and al. (éds.), Revue Économie et Institutions, Dossier : Proximités et institutions nouveaux éclairages, $n^{\circ}$ 6-7, p. 51-74.

Compagnon D., 2008, « La biodiversité, entre appropriation privée, revendications de souveraineté et coopération internationale ", Développement durable \& territoires (en ligne), Dossier 10 : Biens communs et propriété, mis en ligne le 7 mars 2008, consulté le 11 novembre 2011 (URL : http://developpementdurable.revues.org/5253).

Defert P., 1982, « Un nouvel ensemble de ressources touristiques : le mnémôme », Tourism Review, vol. $37, \mathrm{n}^{\circ} 1$, p. $16-18$.

Di Méo G., 2001, Géographie sociale et territoires, Paris, Nathan Université, 317 p.

Doumenge, F., 1994, « Unité et diversité des caractères naturels des îles tropicales », in CRETCEGET (eds.), Nature et hommes dans les îles tropicales : réflexions et exemples, Bordeaux, CRET éditions, p. 9-24. 
Doumenge F., 1987, « Quelques contraintes du milieu insulaire », in J.P. Doumenge and al. (eds.), Iles tropicales : insularité, insularisme, Bordeaux, CRET éditions, p. 9-16.

Edouard J.A., Laune P., 1995, Réserve naturelle de la presqu'île de la Caravelle, Fort-de-France, Parc Naturel Régional de la Martinique, $58 \mathrm{p}$.

Fortier A., Alphandéry P., 2005, « Négociations autour de la biodiversité : la mise en œuvre de Natura 2000 en France », in P. Marty and al. (eds.), Les biodiversités, Paris, CNRS éditions, p. $227-240$.

François H., Hirczak M., Senil N., 2006, « Territoire et patrimoine : la co-construction d'une dynamique et de ses ressources ", Revue d'Économie Régionale \& Urbaine, n 5, p. 683-700.

Grenier C., 2002, « Océaniques ou américaines? Analyse comparative du rattachement à l'Amérique des Galápagos et de l'île de Pâques ", Mappemonde, n 66, p. 38-44.

Grenier C., 1996, Réseaux contre nature, conservation, tourisme et migration aux îles Galápagos (Équateur), Paris, Université Panthéon-Sorbonne, 766 p.

Lacoste A., Salanon R., 2001, Éléments de biogéographie et d'écologie, Paris, Nathan, 318 p.

Lamy M., 1999, La biosphère, la biodiversité et l'homme, Paris, Ellipses, 191 p.

Landel P.A., Senil N., 2009, « Patrimoine et territoire, les nouvelles ressources du développement », Développement durable \& territoires (en ligne), Dossier 12 : Identités, patrimoines collectifs et développement soutenable, mis en ligne le 20 janvier 2009, consulté le 11 novembre 2011 (URL : http://developpementdurable.revues.org/7563).

Larrère R., 2005, « Les considérations éthiques qui justifient la préservation de la biodiversité », in P. Marty and al. (eds.), Les biodiversités, Paris, CNRS éditions, p. 190-193

Le Danff J.P., 2002, « La convention sur la diversité biologique : tentative de bilan depuis Rio », Vertigo (en ligne), vol. 3, n³, 13 novembre 2011, 4 p. (URL : http://vertigo.revues.org/4168).

Leakey R., Lewin R., 1998, La sixième extinction, Paris, Flammarion, 344 p.

Lepart J., 2005, « Diversité et fonctionnement des écosystèmes et des paysages », in P. Marty and al. (eds.), Les biodiversités, Paris, CNRS éditions, p. 83-96.

Lévêque C., Mounolou J.C., 2001, Biodiversité, Paris, Dunod, 248 p.

Lozato-Giotart J.P., 2003, Géographie du tourisme : de l'espace consommé à l'espace maîtrisé, Paris, Pearson Éducation, 330 p.

MacArthur R., Wilson E., 1967, The theory of island biogeography, New Jersey, Princeton University Press, 203 p.

Maljean-Dubois S., 2005, « La biodiversité dans les négociations internationales : de la Convention de Rio sur la diversité biologique au Protocole de Carthagène sur la biosécurité », in P. Marty and al. (éds.), Les biodiversités, Paris, CNRS éditions, p. 211-226.

Marty P., Vivien F.D., Lepart J., Larrère R., 2005, Les biodiversités, Paris, CNRS éditions, 261 p.

Micoud A., 2005, « Comment, en sociologie, tenter de rendre compte de l'émergence du thème de la biodiversité ? », in P. Marty and al. (éds.), Les biodiversités, Paris, CNRS éditions, p. 57-66.

Mittermeier R.A., Gil P.R., Hoffman M., Pilgrim J., Brooks T., Mittermeier C.G., Lamoreux J., Da Fonseca G., 2005, Hotspots Revisited, Conservation International, 391 p.

Myers N., Mittermeier R., Mittermeier C., Da Fonseca G., Kent J., 2000, "Biodiversity hotspots for conservation priorities", Nature, n 403, 24 février 2000, 6 p. (URL : http://www.nature.com). 
Nicolas T., 2005, «L'hypo-insularité, une nouvelle condition insulaire : l'exemple des Antilles françaises », Espace géographique, $\mathrm{n}^{\circ}$ 4, p. 329-34.

OCDE, 2001, Du bien-être des nations : le rôle du capital humain et social, Organisation de Coopération et de Développement Économique, $140 \mathrm{p}$.

ONU, 1992, "Convention on biological diversity", Treaty series, n 1760, 11 août 2009, 307 p. (URL : http://www.cbd.int).

Pecqueur B., 2002, « Dans quelles conditions les objets patrimoniaux peuvent-ils être support d'activités?», Montagnes Méditerranéennes, n 15, p. 123-127.

Pecqueur B., 2001, «Qualité et développement territorial : l'hypothèse du panier de biens et de services territorialisés ", Économie Rurale, n²61, p. 37-49.

Pinchon R., 1976, Faune des Antilles françaises : les oiseaux, Fort-de-France, documentation de la Réserve du Père Pinchon, 326 p.

PNUE, 2002, L'avenir de l'environnement mondial 3, GEO-3, Paris, De Boeck Université, 416 p.

Prescott-Allen R., 2003, Le bien-être des nations, Paris, Éditions ESKA, 368 p.

Ramade F., 2002, Dictionnaire encyclopédique de l'écologie et des sciences de l'environnement. Paris, Dunod, $1075 \mathrm{p}$.

Ricklefs R., Bermingham E., 2004, "History and the species-area relationship in Lesser Antillean birds", The American Naturalist, vol. 163, n², 12 p. (URL : http://www.journals.uchicago.edu).

Ricklefs R., Bermingham E., 2002, "The concept of the taxon cycle in biogeography", Global Ecology et Biogeography, vol. 11, 8 p. (URL : http://www.blackwell-science.com).

Ricklefs R., Cox G., 1972, “Taxon Cycles in the West Indian Avifauna”, The American Naturalist, vol. 106, n 948, 24 p. (URL : http://www.jstor.org).

Selmi A., 2005, « Savoirs naturalistes scientifiques et appropriation du territoire en Vanoise (1963-1990) », in P. Marty and al. (éds.), Les biodiversités, Paris, CNRS éditions, p. 241-258.

Simon L., 2007, « Biodiversité », in Y. Veyret and al. (eds.), Dictionnaire de l'environnement, Paris, Armand Colin, p. 39-40.

Taglioni F., 2003, Recherches sur les petits espaces insulaires et sur leurs organisations régionales, Paris, Université Paris IV-Sorbonne, $218 \mathrm{p}$.

Teyssèdre A., 2005, « Vers une sixième grande crise d'extinctions ?» in R. Barbault and al. (éds.), Biodiversité, science et gouvernance, Paris, ADPF, p. 24-49.

Veyret Y., 2007, Dictionnaire de l'environnement, Paris, Armand Colin, 403 p.

Vivien, F.D., 2005, « La diversité biologique entre valeurs, évaluations et valorisations économiques ", in P. Marty and al. (eds.), Les biodiversités, Paris, CNRS éditions, p. 125-140

Westercamp D., Tazieff H., 1980, Martinique, Guadeloupe, Saint-Martin, la Désirade, Paris, Masson éditeur, $135 \mathrm{p}$.

Whittaker R., 1998, Island Biogeography, New York, Oxford University Press, 277 p.

Wilson E.O., 1988, BioDiversity, Washington, National Academy Press, 496 p. 


\section{NOTES}

1. Le qualificatif biocénotique (ou biocœnotique) renvoie à la notion de biocénose (ou biocœnose), soit la totalité des êtres vivants qui peuplent un écosystème donné (Ramade, 2002).

2. Les régions biogéographiques (ou empires biogéographiques) sont des subdivisions biogéographiques primordiales de la biosphère, correspondant à des sous-continents entiers (Ramade, 2002). Les huit régions communément admises sont le Paléarctique, l'Ethiopien, l'Indomalais, l'Australien, l'Océanien, le Néarctique, le Néotropical et l'Antarctique.

3. Les vertébrés considérés sont les oiseaux, les mammifères, les reptiles et les amphibiens.

4. Le taux d'endémisme se rapporte au nombre d'espèces de vertébrés pour chacune des régions.

5. Traduction de «biological diversity encompasses all species of plants, animals, and microorganisms and the ecosystems and ecological processes of which they are parts " dans MCNEELY, J. A. et al. (1990). Conserving the world's biological diversity. Gland, Switzerland and Washington D.C. : The International Union for Conservation of Nature and Natural Resources, World Resources Institute, Conservation International, World Wildlife Fund-US and World Bank, 193 p., p. 17.

6. Cette expression s'utilise dans les Antilles françaises pour désigner les plus petites îles.

7. Le néologisme biospatial est utilisé pour qualifier un territoire pour lequel un enjeu de la biodiversité a été identifié.

\section{RÉSUMÉS}

L'accroissement des besoins en espace et en ressource naturelle menace l'existence de l'actuelle biosphère par d'irrémédiables changements dans la composition et la structuration de sa diversité biologique. L'état des lieux des relations entre la société et la biodiversité fait ressortir l'extinction d'un certain nombre d'espèces. Face aux effets irréversibles de la chasse et de la destruction de biotopes, l'aménagement et le développement économique des territoires insulaires tendent à se concevoir en intégrant la protection, la conservation et la valorisation de la biodiversité mondiale. Ainsi, en prenant l'exemple des espèces aviennes à la Martinique, la diversité biologique tend à se concevoir de plus en plus comme étant une richesse à conserver mais bien plus encore comme un prétexte dans la mise en protection et dans la valorisation d'un certain nombre d'entités biospatiales, notamment à des fins ludiques.

The increase in the needs for space and natural resource threatens the existence of the current biosphere with incurable changes in the composition and the structuring of its biological diversity. The inventory of the relations between man and biodiversity emphasizes the extinction of a certain number of species specific. Facing the irreversible effects of hunting and the destruction of biotopes, land-use planning and economic development of the insulars territory tend to be conceived by taking into account sustainable management integrating world diversity : that is the biodiversity in its broadest meaning. Thus, the avian species of Martinique are conceived as biological entities to be preserved but much more still like pretexts in the protection and valorization of some biospatials unit, particularly for recreation purposes. 
INDEX

Mots-clés : biodiversité, diversité biologique, Martinique, développement insulaire, avifaune, gestion, ressource, protection/conservation de la nature, valorisation de la nature

Keywords : biodiversity, biological diversity, Martinique, island development, avifauna, management, resource, conservation, valorization

\section{AUTEUR}

\section{JEAN-RAPHAËL GROS-DÉSORMEAUX}

Jean-Raphaël Gros-Désormeaux est post-doctorant en géographe au laboratoire de recherche AIHP-GEODE Caraïbe (EA929) de l'Université des Antilles et de la Guyane et membre de l'UMR ESPACE-DEV (UMR228). Actuellement, il assure la coordination adjointe du projet CARIBSAT à l'Institut de Recherche pour le Développement. jrmgrosdesormeaux@yahoo.fr 